\title{
Harnessing wave power in open seas
}

\author{
Michael Stiassnie $^{1}$ - Usama Kadri ${ }^{2}$ - Raphael Stuhlmeier ${ }^{1}$
}

Received: 9 March 2015 / Accepted: 11 October 2015 / Published online: 5 November 2015

(C) Springer International Publishing Switzerland 2015

\begin{abstract}
We discuss some of the latent potential for harnessing wave power in open seas. Large farms of waveenergy converters in the open sea may extract energy several times over the course of an ocean basin, allowing the waves to grow under the influence of the wind, and capturing energy otherwise dissipated. Our calculations show that such an approach results in increasing the wave power potential by an order of magnitude compared to coastal capture alone. To carry out such calculations one needs the captured, reflected, and transmitted energy transfer functions of the farms. Here we simulate the functioning of the farms by one simplified two-dimensional converter consisting of two vertical floating plates, for which explicit transfer functions are calculated. Our main goals are to increase the awareness of the scientific community to the importance of harvesting wave power in open seas, and to provide a preliminary picture for the geometry and size of wave energy farms in open seas.
\end{abstract}

Keywords Wave energy - Wave-energy converter . Wave-structure interaction · Wind waves · Open seas

Michael Stiassnie

miky@technion.ac.il

Usama Kadri

usama.kadri@gmail.com

Raphael Stuhlmeier

raphaels@technion.ac.il

1 Faculty of Civil and Environmental Engineering, Technion-Israel Institute of Technology, 32000 Haifa, Israel

2 Department of Mechanical Engineering, Massachusetts Institute of Technology, Cambridge, MA 02139, USA

\section{Introduction}

The remarkable property of water waves, once created by the wind, to propagate energy over very long distances with little loss has naturally given rise to the desire to harness this energy and convert it to a useful form. The promise of this form of renewable power is clear: winds transfer energy into wave motion at a rate of about $5.6 \times 10^{13} \mathrm{~W}$, which is comparable to the world's present power consumption of about $1.5 \times 10^{13} \mathrm{~W}$ (see Rascle et al. 2008; Rascle and Ardhuin 2013). Some fraction thereof is exploitable in practice, but the burgeoning state of wave-energy conversion makes this hard to quantify (see e.g., Cruz 2008 or Multon 2011).

Many challenges await in the development of large-scale, commercial wave power. These are connected both with assessing the resource variability, selecting proper sites, as well as aspects of the devices used-from the hydrodynamics, to survivability and power take-off-to undersea-cables and grid connection. Recent reviews of many of these aspects may be found in the books by Cruz (2008) and Multon (2011), the influential article by Falcão (2010), or the report Magagna et al. (2014) by the EU-funded Strategic Initiative for Ocean Energy.

It is clear that the economic feasibility of wave energy requires the development of large-scale converters or converter arrays (suggestively termed wave farms), and likely that progress in offshore devices (so-called third-generation devices) will continue to grow. The basic rationale behind this move may be illustrated by considering the power input which goes into wave generation, of which $96 \%$ is lost to wave breaking in open seas, and only $2.5 \times 10^{12} \mathrm{~W}$ (i.e., $4 \%$ ) is dissipated in the surf of coastal waters (Rascle and Ardhuin 2013).

It is our contention that the long-term future of waveenergy extraction will involve a move further offshore, into 
the deep waters of the open oceans. In addition to exploiting the more powerful wave regimes in deep water, this also allows for the possibility of capturing energy multiple times over an expanse of ocean, rather than once in the vicinity of shore. The wave field may thus be allowed to regrow under the influence of the wind between one device (or device-array) and the next. The 20-fold difference between energy lost through wave breaking on the open seas and that lost on our coasts illustrates the potential for such a concept in increasing markedly the percentage of exploitable wave energy.

In this paper, we attempt to make a first, preliminary step in this direction. Restricting ourselves to one-dimensional propagation, we first review the concept of fetch-limited wave growth in Sect. 2, and make several simple observations about energy capture by an ideal wave farm which captures the totality of the incoming wave energy. Despite the multitude of proposed wave-energy devices, most are confined to the near shore, and the vast majority do not readily yield values of transmission and reflection coefficients needed for our theoretical study. Thus, in Sect. 3 we develop an illustrative wave-energy converter based on two interconnected, vertically floating plates, which we use as a simple representation of a generic, semi-infinite wave farm. In Sect. 4 we apply this model to illustrate the harvesting of wave energy in the open ocean-particularly the potential for extraction, regrowth, and re-extraction over the length of a ocean basin. A discussion of the results, considering also avenues for future research is given in Sect. 5, while some mathematical coefficients and properties of the device appear in Appendices 1 and 2.

\section{Fetch-limited wave growth}

\subsection{The JONSWAP spectrum}

Fetch-limited growth occurs when a wind of constant magnitude blows perpendicular to a long and straight coastline. The water is assumed deep and the wind blows for a sufficiently long time that the wavefield reaches a steady state. Hence, for a given wind speed, the wavefield becomes a function only of the distance from the shoreline, which is termed the fetch.

The case of fetch-limited growth has been extensively investigated both by laboratory and field measurements. Here we adopt the approach of Holthuijsen (2008) and assume that these wave fields can be represented by one-dimensional JONSWAP spectra, as given by Hasselmann et al. (1973). For long-enough fetches, these wave fields become fully developed, and the JONSWAP spectra evolve into PiersonMoskowitz spectra, see Pierson and Moskowitz (1964).

We denote the energy spectra by $E$, the frequency by $\omega$ and fetch by $x$, and their dimensionless forms by $\tilde{E}=$ $E g^{2} \rho^{-1} U_{10}^{-5}, \tilde{\omega}=\omega U_{10} g^{-1}$, and $\tilde{x}=x g U_{10}^{-2}$, where $U_{10}$ is the wind speed at $10 \mathrm{~m}$ above the mean sea surface, $\rho$ is the density of water, and $g$ is gravity.

In terms of these dimensionless variables, the JONSWAP spectra are written as

$\tilde{E}=\tilde{\alpha} \tilde{\omega}^{-5} \exp \left[-\frac{5}{4}\left(\frac{\tilde{\omega}}{\tilde{\omega}_{p}}\right)^{-4}\right] \tilde{\gamma}^{\exp \left(-\frac{1}{2}\left(\frac{\tilde{\omega} / \tilde{\omega}_{p}-1}{\tilde{\sigma}}\right)^{2}\right)}$

where the peak frequency

$\tilde{\omega}_{p}= \begin{cases}13.7 \tilde{x}^{-0.27} & \tilde{x} \leq 3.44 \times 10^{4} \\ 0.816 & \tilde{x} \geq 3.44 \times 10^{4}\end{cases}$

and

$\tilde{\alpha}=0.00926 \tilde{\omega}_{p}^{0.67}$
$\tilde{\gamma}=1.21 \tilde{\omega}_{p}^{0.86}$
$\tilde{\sigma}= \begin{cases}0.030 \tilde{\omega}_{p}^{0.32} & \tilde{\omega}<\tilde{\omega}_{p} \\ 0.058 \tilde{\omega}_{p}^{0.16} & \tilde{\omega}>\tilde{\omega}_{p}\end{cases}$

Note that the dimensionless fetch for which the sea becomes fully developed, and beyond which the energy that is transfered from the wind to the waves is completely lost due to breaking, is $\tilde{x}=3.44 \times 10^{4}$.

We will also make use of the result due to Plant (1982), based on Miles' (1957) wave growth mechanism, namely that the energy input from the wind to a wave with frequency $\tilde{\omega}$ is governed by the equation

$\frac{\mathrm{d} \tilde{E}}{\mathrm{~d} \tilde{x}}=2.5 \times 10^{-4} \tilde{\omega}^{4} \tilde{E}$.

\subsection{An ideal wave power harvesting farm}

We apply the above concepts to discuss briefly the behavior of an ideal, perfect wave power farm, which is assumed to capture the entire energy of the incoming waves. Given this crude, ideal model, in what follows we shall be concerned solely with obtaining parameters for the layout of these farms, primarily their spacing over an ocean basin for varying wind conditions, and the resulting potential for increasing the captured power.

The total energy flux per unit width in $\mathrm{W} / \mathrm{m}$, as a function of wind $U_{10}$ and fetch $x$ is derived from the JONSWAP spectrum via

$F\left(U_{10}, x\right)=\int_{0}^{\infty} E \cdot c_{g} \mathrm{~d} \omega$

where $c_{g}=\mathrm{d} \omega / \mathrm{d} k$ is the group velocity, for the wave number $k=\omega^{2} / g$. Here and subsequently, we shall choose a design wind of $10 \mathrm{~m} / \mathrm{s}\left(U_{10}\right)$, and denote variables for the 
'design conditions' by a subscript d. Weaker and stronger winds are taken as 7.5 and $12.5 \mathrm{~m} / \mathrm{s}$, respectively, and associated variables are denoted by the addition of superscripts and + , respectively. Table 1 depicts the fetch $f$ needed for a fully developed sea for these different wind speeds, and the energy flux associated with this fully developed sea state in each case.

To illustrate the advantages of multiple such farms in the open ocean, we assume a schematic basin (see Fig. 1) of length $L 2000 \mathrm{~km}$. With the design wind $U_{\mathrm{d}}=10 \mathrm{~m} / \mathrm{s}$ blowing over the basin, Table 1 shows that the energy spectrum of the sea state will reach saturation after $f_{d}=350.7 \mathrm{~km}$. Note that the total energy flux grows slightly faster than linearly with fetch.

It is thus optimal to place five perfect wave farms at intervals of length $\Delta d=f_{d}$ along the basin, with a leading fetch $x_{0}=2000-4 \times \Delta d=597 \mathrm{~km}$, which determines the design geometry. This allows for the capture of five times the energy flux of the fully developed state $F\left(U_{\mathrm{d}}, f_{d}\right)$ (see Fig. 1 for the schematic geometry; the captured flux is given per meter of breadth $B$ ).

The performance of this design geometry is now evaluated for different wind speeds. From Table 1, it is seen that the fetch needed for full development under weaker and stronger winds is $f_{d}^{-}=197.2 \mathrm{~km}$ and $f_{d}^{+}=547.9 \mathrm{~km}$, respectively. As $f_{d}^{-}<\Delta d$ it is clear that the total captured flux for the lesser wind speed is five times the total flux captured by one such absorber.

Conversely, as $f_{d}^{+}>\Delta d$, the sea state between the farms does not return to the fully developed sea. Consequently, the total captured flux is $F\left(U_{\mathrm{d}}^{+}, x_{0}\right)+4 \times F\left(U_{\mathrm{d}}^{+}, \Delta d\right)$, i.e., the

Table 1 Fetch needed for full development, and total flux, as functions of wind speeds

\begin{tabular}{lll}
\hline$U_{10}(\mathrm{~m} / \mathrm{s})$ & $f(\mathrm{~km})$ & $F\left(U_{10}, f\right)(\mathrm{KW} / \mathrm{m})$ \\
\hline 7.5 & 197.2 & 4.6 \\
10 & 350.7 & 19.9 \\
12.5 & 547.9 & 59.7 \\
\hline
\end{tabular}

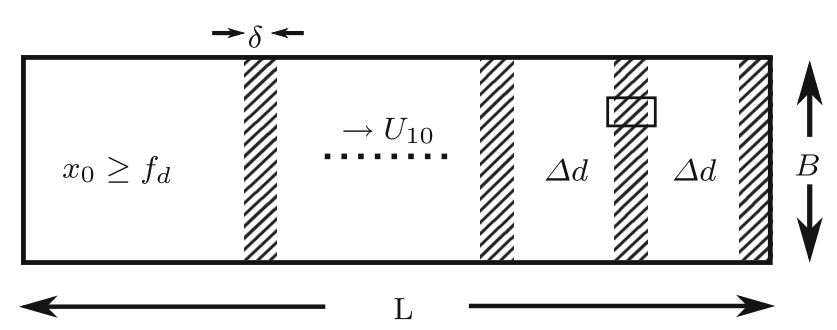

Fig. 1 Schematic geometry of an ocean basin of length $L$ with a number of wave farms (shaded areas) a distance $\Delta d$ apart. A constant wind blows from the left, and $x_{0}$ indicates the leading fetch prior to the first device. $\delta$ is the farm width, and $B$ the harvesting breadth orthogonal to the wind direction
Table 2 Total energy flux captured by 5 perfect wave farms spaced a distance $f_{d}=350.7 \mathrm{~km}$ apart over an ocean basin with $L=2000 \mathrm{~km}$, for different wind speeds

\begin{tabular}{lc}
\hline$U_{10}(\mathrm{~m} / \mathrm{s})$ & Total captured energy flux $(\mathrm{KW} / \mathrm{m})$ \\
\hline 7.5 & 23.2 \\
10 & 99.5 \\
12.5 & 212.5 \\
\hline
\end{tabular}

flux available after a leading fetch of $x_{0}$ added to the flux captured by the subsequent four perfectly absorbing farms for a wind $U_{\mathrm{d}}^{+}$blowing over a fetch of $\Delta d=350.7 \mathrm{~km}$ (see Fig. 1). Since $597.3 \mathrm{~km}=x_{0}>f_{d}^{+}$, the captured flux by the first farm is $59.7 \mathrm{KW} / \mathrm{m}$. The subsequent contributions amount to an additional $152.8 \mathrm{KW} / \mathrm{m}$ for a total of $212.5 \mathrm{KW} / \mathrm{m}$. These results are summarized in Table 2.

The values of the total energy fluxes given in Table 2 should be compared with those for coastal capture as given in Table 1, resulting in a fivefold increase in the power capturing potential for $U_{\mathrm{d}}$ and $U_{\mathrm{d}}^{-}$, and a 3.5 -fold increase for $U_{\mathrm{d}}^{+}$. While such perfect farms may be unrealistic in practice, for regular beam-seas (a 2D configuration) perfect absorption is theoretically possible by optimizing the device design (see Falnes 2007), for a broadband beam-sea state it requires also active control. In addition, some theoretical results such as Salter's duck, or the dense arrays of small buoys presented by Garnaud and Mei (2009) yield comparable efficiencies (98\% absorption for $U_{\mathrm{d}}=10 \mathrm{~m} / \mathrm{s}$ ) for a water depth $h=100 \mathrm{~m}$, and a semi-infinite square array consisting of 26 columns of buoys of draft and radius $5 \mathrm{~m}$, spaced sequentially over a farm width of $\delta=500 \mathrm{~m}$.

\subsection{A vision for future open-sea wave power farms}

The ideal farm above serves to illustrate an important point: as the wave field downwind of any wave farm will be modified by it, situating such a farm in the open sea allows for the wave field to grow further under the influence of the wind, and invites the possibility of capturing energy multiple times over the course of an ocean basin. This approach provides a different conception of the wave-energy resource. Rather than concentrating upon a one-dimensional curve of coastline which may promise some average power in $\mathrm{KW} / \mathrm{m}$ for a stretch of time, we may conceive of a two-dimensional resource that may be tapped in the future by series of arrays.

Current wave farms are for the most part in prototype or planning stages - the world's first wave farm, in Aguçadoura, Portugal, consisted of only three Pelamis devices and operated for two months beginning in September 2008. The Perth Wave Energy Project, in Australia, while operational as of 2014 with two Carnegie CETO buoys, is for the moment a commercial-scale demonstration, albeit grid connected. 


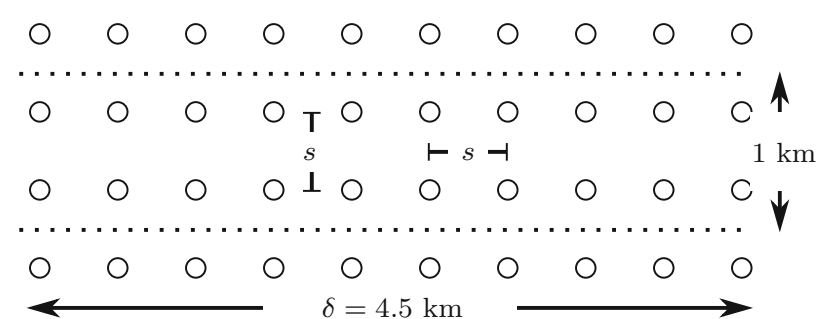

Fig. 2 Schematic picture of part of a wave farm, as a rectangular array with spacing $s=500 \mathrm{~m}$, and 10 units deep in the direction of wave propagation. This figure may be considered to depict the small rectangular region of Fig. 1

Other projects, such as Wave Hub in South West England, are at present in various stages of development.

It is to be expected that future developments will lead to much larger wave farms. As an example, for a rectangular array consisting of ten wave-energy absorbers per row (see Fig. 2), with a spacing $s=500 \mathrm{~m}$, one obtains an array width $\delta=4.5 \mathrm{~km}$ in Fig. 1, with 20 units per kilometer of $B$, where $B$ may be in the order of $100 \mathrm{~km}$. At the moment, no results exist on energy capture, reflection, and transmission of such large-scale, sparse (i.e., $k s \gg 1$ ) arrays in deep water $(k h \gg 1)$.

The simplistic example of the ideal farm above already provides an indication of the potential for harnessing wave energy in the open ocean, and our use of the JONSWAP spectrum gives an order of magnitude estimate for the spacing between farms. In what follows, we will move to a more realistic configuration-using a structure of two vertically floating plates to yield frequency-dependent transfer functions, which are used to model such a farm.

\section{Derivation of the transfer functions for two vertically floating plates}

\subsection{Formulation of the mathematical problem}

The proposed model is composed of two vertically floating identical plates interconnected through hinges by means of two horizontal bars with dampers, see Fig. 3, and allowed to sway and roll. We consider a two-dimensional model in the $(x, z)$ plane. The $x$-axis coincides with the undisturbed water surface and the $z$-axis points downwards. We assume irrotational flow in infinitely deep water, and remain within the framework of linear wave theory. Thus, the governing equation and free-surface boundary conditions are

$$
\begin{aligned}
\phi_{, x x}+\phi_{, z z} & =0, \quad z \geq 0 \\
\phi_{, \tau \tau}-g \phi_{, z} & =0, \quad z=0,
\end{aligned}
$$

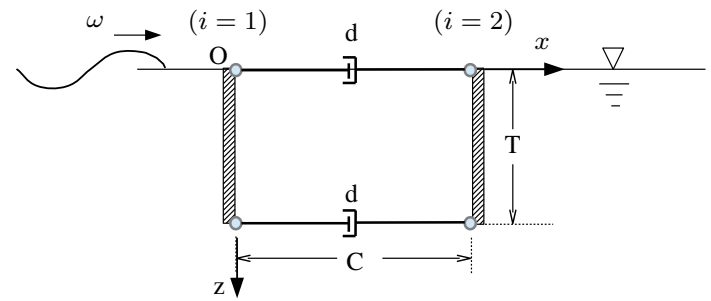

Fig. 3 A sketch of the model. $T$ Draft, $C$ spacing, $d$ damping coefficient per unit width divided by the density of water

where $\phi$ is the velocity potential, $\tau$ is the time, and the subscripts preceded by a comma denote partial differentiation.

The potential of an incident wave, with frequency $\omega$, and unit amplitude, approaching from the left $(x=-\infty)$ is given by

$\phi_{0}=-\frac{j g}{\omega} \exp [j \omega \tau-k(z+j x)]$,

with $k=\omega^{2} / g, j=\sqrt{-1}$.

The boundary conditions on the plates (1) and (2) (after linearisation) are

$$
\begin{aligned}
& \phi_{, x}=j \omega\left(H_{1}+A_{1} z\right), \quad x=0 \text { and } 0<z<T, \\
& \phi_{, x}=j \omega\left(H_{2}+A_{2} z\right), \quad x=C \text { and } 0<z<T .
\end{aligned}
$$

$H_{1}$ and $H_{2}$ are the ratios between the horizontal displacement amplitudes of the points $(0,0)$ and $(C, 0)$ and the incident wave amplitude, respectively. $A_{1}$ and $A_{2}$ are the amplitudes of the angular motions of the plate about $(0,0)$ and $(C, 0)$ per unit wave amplitude, respectively.

The solution of the mathematical problem is rendered unique with the help of the so-called radiation conditions at $x \rightarrow \pm \infty$.

In Sect. 3.2, we summarize the closed mathematical solution for a single plate as given by Haskind (1959). An experimental verification of Haskind's solution is given by Stiassnie et al. (1981). In Sect. 3.3, we utilise the wide spacing assumption, see Srokosz and Evans (1979), to obtain a formulation in the form of a linear algebraic system of equations. Section 3.4 contains energy balance considerations.

\subsection{Haskind's solution for a single plate}

The case of a single plate has been studied by numerous investigators [notably Ursell (1947, 1948), and in the context of floating breakwaters by Stiassnie (1980)]. The following is based on results of Haskind (1959). The transmission coefficient $T_{\mathrm{t}}$ (defined as the ratio of transmitted to incident wave amplitude) for a thin plate, submerged to a depth $T$, is given by 
$T_{\mathrm{t}}=t+B_{2} H+B_{4} A$,

where $t$ is the transmission coefficient of a rigidly held plate; $H$ is the ratio between the horizontal displacement amplitude of the point $O$ (intersection between the plate and the undisturbed water surface) and the incident wave amplitude; $A$ is the amplitude of the angular motion about $O$ per unit wave amplitude. For the case of a weightless freely floating plate, the expressions for $H$ and $A$ are:

$H=\left(-Y_{g} D_{44}+M_{g} D_{24}\right) / D$

$A=\left(Y_{g} D_{42}-M_{g} D_{22}\right) / D$.

The physical meaning of each of the various quantities in Eqs. (13) and (14) is as follows: $B_{2}, B_{4}$ are the amplitudes of the waves radiated in the positive direction by a unit amplitude of horizontal and angular displacements, respectively, for a single plate; $Y_{g}, M_{g}$ are the force and moment exerted on the plate by a unit amplitude wave arriving from the left. We also have

$D_{p q}=\omega^{2} \mu_{p q}-j \omega \lambda_{p q} \quad(p$ and $q=2,4)$;

$D=D_{22} D_{44}-D_{24} D_{42}$,

where $\mu_{p q}$ are the added mass coefficients and $\lambda_{p q}$ are the damping coefficients. As in Srokosz and Evans' (1979) treatment of two thin barriers, here too the mass and moment of inertia are negligible compared with the added mass and damping due to the fluid. The indices 2 and 4 refer to horizontal and angular motions, respectively. The detailed mathematical expressions for the above-mentioned quantities are rather long and are given in Appendix 1.

\subsection{Solution for twin plates}

The wide spacing assumption means that the plates are spaced far enough from one another, so that the local wave field in the vicinity of one plate (i.e., the evanescent part) does not influence the other. The only interaction between the plates is due to the far field propagating wave terms which appear in the radiation and scattering problems for a single plate. Let us observe now two identical plates as shown in Fig. 4. The terms $R_{1}, R_{2}$ denote the amplitudes of the waves traveling to the right. The amplitudes of the waves traveling to the left are denoted by $L_{1}, L_{2}$. The subscript $i=1,2$ indicates that the wave approaches the $i$ th plate (either from its right or left side). Without loss of generality the incident wave from the left was considered to have a unit amplitude, i.e., $R_{1}=1$. The parameter $L_{2}$ was set equal to zero in accordance with Sommerfeld's radiation condition. As mentioned in Sect. 2.1, we denote the horizontal displacements of the intersection points of the plates $i=1,2$ and the undisturbed water surface by $H_{1}, H_{2}$. The quantities $A_{1}, A_{2}$ are

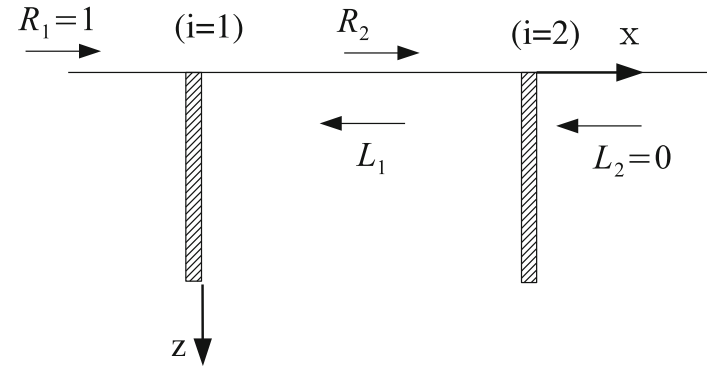

Fig. 4 Definition of unknowns

the amplitudes of the angular motion about these intersection points.

$H_{1}, H_{2}, A_{1}, A_{2}, R_{2}$, and $L_{1}$ constitute the six unknown variables of the problem, whereas $\omega, T, C$, and $d$ are given parameters. Neglecting the mass of the plates reduces their equations of horizontal/angular motion to balances of forces/moments. The balances of horizontal forces, for plates 1,2 , respectively, gives

$$
\begin{aligned}
& D_{22} H_{1}+D_{24} A_{1}+Y_{g}\left(R_{1}-L_{1}\right)-2 j \omega d\left(H_{1}-H_{2}\right) \\
& \quad-j \omega T d\left(A_{1}-A_{2}\right)=0, \\
& \quad D_{22} H_{2}+D_{24} A_{2}+Y_{g} R_{2}+2 j \omega d\left(H_{1}-H_{2}\right) \\
& \quad+j \omega T d\left(A_{1}-A_{2}\right)=0 .
\end{aligned}
$$

The balances of moments, for plates 1,2 are, respectively,

$$
\begin{aligned}
& D_{24} H_{1}+D_{44} A_{1}+M_{g}\left(R_{1}-L_{1}\right)-j \omega T d\left(H_{1}-H_{2}\right) \\
& \quad-j \omega T^{2} d\left(A_{1}-A_{2}\right)=0, \\
& D_{24} H_{2}+D_{44} A_{2}+M_{g} R_{2}+j \omega T d\left(H_{1}-H_{2}\right) \\
& \quad+j \omega T^{2} d\left(A_{1}-A_{2}\right)=0 .
\end{aligned}
$$

A consequence of the wide spacing assumption is that the far field to the right of plate 1 is identical with the far field to the left of plate 2 . In this way we obtain an additional pair of equations, one for the wave propagating in the positive direction and the other for the wave propagating in the negative direction:

$$
\begin{gathered}
B_{2} H_{1}+B_{4} A_{1}+t R_{1}+r L_{1}=E R_{2}, \\
-B_{2} H_{2}-B_{4} A_{2}+r R_{2}=E L_{1},
\end{gathered}
$$

where $r$ is the reflection coefficient for a rigidly held single plate and $E=\exp (j k C)$. Equations (16)-(21) constitute together a linear algebraic system of six equations with the same number of unknowns.

\subsection{Energy balance}

Neglecting energy dissipation due to vortex shedding from the lower edges of the plates (see Stiassnie et al. 1984), the 
(time averaged) incoming energy flux, $F_{\mathrm{i}}$, should equal the sum of the reflected energy flux $F_{\mathrm{r}}$, the transmitted energy flux $F_{\mathrm{t}}$, and the power absorbed by the dampers $P_{\mathrm{a}}$ :

$F_{\mathrm{i}}=F_{\mathrm{r}}+F_{\mathrm{t}}+P_{\mathrm{a}}$.

The fluxes are given by multiplying the energy densities by the group velocity $c_{g}=0.5 \omega / k$; which gives, for $R_{1}=1$,

$F_{\mathrm{i}}=\left(\frac{1}{2} \rho g\right) c_{g}$

$F_{\mathrm{r}}=\left(\frac{1}{2} \rho g\right)\left|T_{\mathrm{r}}^{2}\right| c_{g}$

and

$F_{\mathrm{t}}=\left(\frac{1}{2} \rho g\right)\left|T_{\mathrm{t}}^{2}\right| c_{g}$,

where the reflection coefficient, $T_{\mathrm{r}}$, and transmission coefficient, $T_{\mathrm{t}}$, for the two-plate system, are

$T_{\mathrm{r}}=r-B_{2} H_{1}-B_{4} A_{1}+t L_{1}$,

$T_{\mathrm{t}}=t R_{2}+B_{2} H_{2}+B_{4} A_{2}$.

The time averaged power that is absorbed by both dampers is

$P_{\mathrm{a}}=\frac{1}{2} \rho \omega^{2} d\left(\left|H_{1}-H_{2}\right|^{2}+\left|H_{1}+T A_{1}-H_{2}-T A_{2}\right|^{2}\right)$.

Note that the relative error in our calculations, given by

$e r=\left|F_{\mathrm{i}}-F_{\mathrm{r}}-F_{\mathrm{t}}-P_{\mathrm{a}}\right| / F_{\mathrm{i}}$,

was always found to be less than $10^{-4}$.

We find a maximum value for dimensionless absorbed power $P_{\mathrm{a}} / F_{\mathrm{i}}$ [see (28)] of 0.5 for nondimensional draft $T_{m}=0.71 k_{\mathrm{p}}^{-1}$, plate spacing $C_{m}=1.05 k_{\mathrm{p}}^{-1}$, and damping $d_{m}=0.88 g^{1 / 2} k_{\mathrm{p}}^{-3 / 2}$, where $k_{\mathrm{p}}$ is the wavenumber to which the system is tuned. This chosen maximum is not uniquefurther details are given in Appendix 2. Note also that this is smaller than what can be attained by a link with a fixed mechanical reference (such as the ground), where a maximum for dimensionless absorbed power of 1 is possible.

\section{Harvesting wave power}

We move now to the harvesting of energy from water waves in the open sea. Irrespective of the design of a given twodimensional device or farm, it may be characterized by transfer functions giving the absorption, reflection, and transmission as functions of wavelength. Although a number

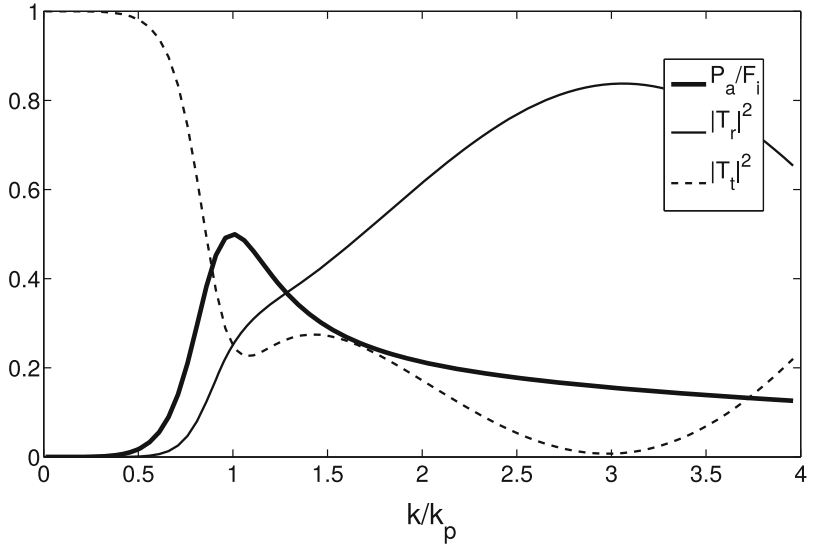

Fig. 5 Transfer functions: power absorption ratio $P_{\mathrm{a}} / F_{\mathrm{i}}$, power reflection ratio $\left|T_{\mathrm{r}}\right|^{2}$, and power transmission ratio $\left|T_{\mathrm{t}}\right|^{2}$ as a function of wavenumber for a device with $k_{\mathrm{p}} T=0.71, k_{\mathrm{p}} C=$ $1.05, g^{-1 / 2} k_{\mathrm{p}}^{3 / 2} d=0.88$

of designs for wave-energy extraction devices have been proposed, explicit transfer functions - in particular transmission coefficients that characterize the energy shadow of the device - are not readily available. Moreover, transfer functions for large farms do not currently appear in the literature, hence those derived in Sect. 3 for the simple device are used to bridge this gap, so as to investigate the potential for harvesting wave power in the open ocean.

Thus, for the purpose of what follows, the relevant parameters are the transfer functions given in Fig. 5-the power absorption ratio $P_{\mathrm{a}} / F_{\mathrm{i}}$, power reflection ratio $\left|T_{\mathrm{r}}\right|^{2}$, and power transmission ratio $\left|T_{\mathrm{t}}\right|^{2}$ as a function of wavenumber versus peak (tuning) wavenumber $k / k_{\mathrm{p}}$. At the peak wavenumber, the absorption rate is $50 \%$, and transmission and reflection each make up $25 \%$. An extended version of this figure may be found in Appendix 2.

As in the case of the perfect wave power farm in Sect. 2.2, we will examine the performance of our simple model in an ocean basin of length $L=2000 \mathrm{~km}$. The design wind $U_{\mathrm{d}}=10 \mathrm{~m} / \mathrm{s}$ and the two test cases $U_{\mathrm{d}}^{+}=12.5 \mathrm{~m} / \mathrm{s}$ and $U_{\mathrm{d}}^{-}=7.5 \mathrm{~m} / \mathrm{s}$ of Sect. 2.2 are retained.

\subsection{Wave power harvesting near the coast}

We first discuss the performance of our simple wave-energy converter, which is tuned to operate optimally for the design wind $U_{\mathrm{d}}$, when this illustrative device is placed at the end of the ocean basin. As the fetch available far exceeds that needed for a fully developed sea, the incoming spectrum is a Pierson-Moskowitz spectrum with peak frequency $\omega_{\mathrm{p}}=$ 0.8005 . This implies a plate draft $T=10.9 \mathrm{~m}$, and a plate spacing $C=16.1 \mathrm{~m}$.

Figure 6 depicts the incoming (fully developed) spectrum at the coastline for the design wind. At the peak of the spec- 


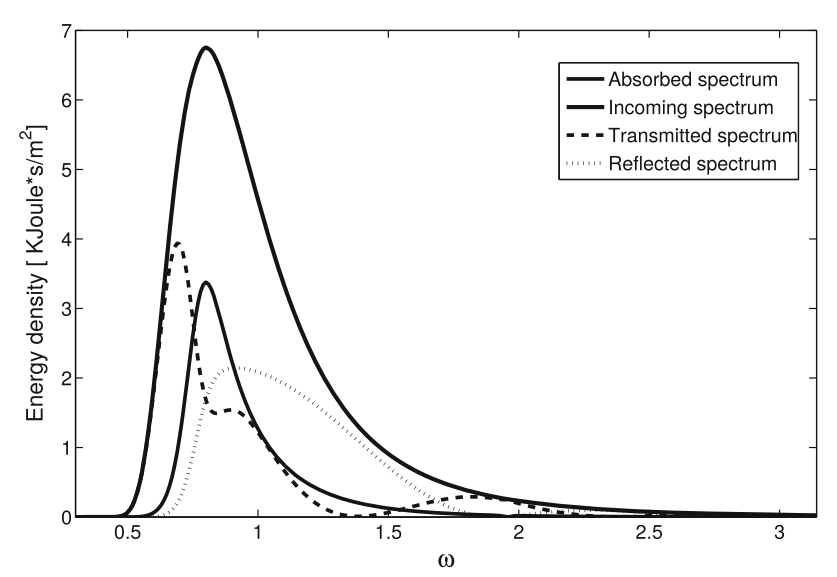

Fig. 6 Pierson-Moskowitz spectrum for a wind $U_{10}=10 \mathrm{~m} / \mathrm{s}$, fetch $2000 \mathrm{~km}$ (thick solid line) and its interaction with a converter. The absorbed component (thin solid line), transmitted component (dashed line), and reflected component (dotted line) are given for a converter tuned for a peak at $\omega_{\mathrm{p}}=0.8005$

Table 3 Energy flux (in $\mathrm{KW} / \mathrm{m}$ ) of Pierson-Moskowitz spectra for constant winds $U_{10}=7.5,10$, and $12.5 \mathrm{~m} / \mathrm{s}$ blowing over a fetch of $2000 \mathrm{~km}$

\begin{tabular}{lrrr}
\hline$U_{10}(\mathrm{~m} / \mathrm{s})$ & 7.5 & 10 & 12.5 \\
\hline Incoming significant wave height $(\mathrm{m})$ & 1.4 & 2.4 & 3.8 \\
Incoming energy flux $(\mathrm{KW} / \mathrm{m})$ & 4.6 & 19.9 & 59.7 \\
Absorbed energy flux $(\mathrm{KW} / \mathrm{m})$ & 1.1 & 5.6 & 11.5 \\
Transmitted energy flux $(\mathrm{KW} / \mathrm{m})$ & 1.0 & 7.6 & 38.6 \\
Reflected energy flux $(\mathrm{KW} / \mathrm{m})$ & 2.5 & 6.4 & 9.5 \\
\hline
\end{tabular}

Energy flux absorbed, transmitted, and reflected by a device designed to operate optimally at $U_{10}=10 \mathrm{~m} / \mathrm{s}$ is given for each case

trum, where the device is most efficient, half the energy is extracted by the device. For other frequencies, the majority of the energy passes to the transmitted/reflected spectra.

In Table 3 we summarize the performance of this converter for an ocean basin with uniform winds $U_{\mathrm{d}}, U_{\mathrm{d}}^{-}$and $U_{\mathrm{d}}^{+}$. In all cases, the available fetch $(2000 \mathrm{~km})$ exceeds that needed for full development. For $U_{\mathrm{d}}^{-}$, the spectral peak is $\omega_{\mathrm{p}}^{-}=1.067$, while for $U_{\mathrm{d}}^{+}$the spectral peak is $\omega_{\mathrm{p}}^{+}=0.6404$, whereas our device is tuned to $\omega_{\mathrm{p}}=0.8005$.

From Table 3 we see that in the design case, slightly less than one-third of the total incoming energy is absorbed by the device. For $U_{\mathrm{d}}^{-}$, the overall efficiency is just less than one quarter, while for $U_{\mathrm{d}}^{+}$it is less than $20 \%$.

\subsection{Wave power harvesting in the open sea}

As has been described previously in Sect. 2.2, we wish to examine the potential benefits of situating future wave farms throughout an ocean basin rather than solely at one end near the coast. For this purpose, the representative device design detailed previously is retained: the device is tuned to operate optimally for the peak frequency of a fully developed sea state with $U_{\mathrm{d}}=10 \mathrm{~m} / \mathrm{s}$.

For this design wind $U_{\mathrm{d}}$, we will allow the sea state to reach energetic saturation (which requires a fetch in excess of $350.7 \mathrm{~km}$ ). Emplacing the first device after sufficient fetch will allow us to extract slightly less than $1 / 3$ of the available energy, while nearly $40 \%$ is transmitted. The reflected component may be neglected, as it propagates counter to the wind.

From the transfer functions (see Fig. 5 and Sect. 3.4), it may be seen that the peak of the transmitted spectrum is one quarter of the peak of the incoming spectrum. We assume that the subsequent growth of the spectrum is governed by Miles' mechanism [see (6)], so that the transmitted spectrum $s_{2}$ after a distance $\Delta d$ is related to the incoming spectrum, denoted $s_{1}$, by

$s_{2}(\omega)=s_{1}(\omega) \cdot\left|T_{\mathrm{t}}(\omega)\right|^{2} \cdot \mathrm{e}^{2 \beta(\omega) \Delta d}$,

where

$\beta(\omega)=1.28 \cdot 10^{-4} \cdot \omega^{4} g^{-3} U_{10}^{2}$.

Note that $T_{\mathrm{t}}$ depends on the frequency $\omega$ (thus on the wavenumber $k$ ) as depicted in Fig. 5 [see also (27) and the coefficients given in Appendix 1]. If we now allow the transmitted spectrum to grow with $U_{10}=U_{\mathrm{d}}$ until its peak energy (at $\omega_{\mathrm{p}}=0.8005$ ) coincides with that of the fully developed sea, we find from (30) $\Delta d=124.51 \mathrm{~km}$. Over this fetch, with the design wind, the transmitted spectrum returns to the fully developed state.

This yields all the parameters needed to give the design configuration over the entire ocean basin: over a fetch of $L=2000 \mathrm{~km}$, we may place 14 wave power converters a distance $\Delta d=124.51 \mathrm{~km}$ apart. This leaves a leading fetch $x_{0}=381.44 \mathrm{~km}$ (see Fig. 1).

We summarize the results for this configuration of 14 converters and the wind speeds $U_{\mathrm{d}}, U_{\mathrm{d}}^{-}$and $U_{\mathrm{d}}^{+}$in Table 4 . In the fields denoting a percentage of coastal capture, the total absorbed power over the 14 emplaced devices is divided by the total absorbed power for a single device placed at the coast (in which instance all incoming spectra are fully developed). The leading fetch $x_{0}$ is sufficient to form a fully developed sea for $U_{\mathrm{d}}$ and $U_{\mathrm{d}}^{-}$, but not for $U_{\mathrm{d}}^{+}$. After each device, the corresponding transmitted spectrum is allowed to grow under influence of the wind according to Miles' mechanism, provided the energy content of any component not exceed that of the fully developed spectrum. For $U_{\mathrm{d}}^{-}$, by (30), a gradual diminution of energy in the lower frequencies is observed over each of the 14 devices, resulting in less than a 14-fold gain in absorbed power versus a single device placed on the coast. In contrast, for $U_{\mathrm{d}}^{+}$the transmitted spectrum grows beyond its value at $x_{0}$, and nearly becomes 
Table 4 Summary of results for 14 sequential converters in a basin of length $2000 \mathrm{~km}$, with different wind speeds

\begin{tabular}{llll}
\hline$U_{10}(\mathrm{~m} / \mathrm{s})$ & 7.5 & 10 & 12.5 \\
\hline Incoming energy flux at $x_{0}(\mathrm{KW} / \mathrm{m})$ & 4.6 & 19.9 & 39.4 \\
Total absorbed power $(\mathrm{KW} / \mathrm{m})$ & 12.2 & 78.3 & 158.9 \\
Percentage of coastal capture $(\%)$ & 1142 & 1400 & 1381 \\
\hline
\end{tabular}

Given is the incoming energy from the leading fetch $x_{0}$, and the total absorbed energy from all 14 devices, as well as the percentage this represents of the single converter installed at the coast

fully developed. This results in somewhat more than a 14fold gain in absorbed power versus the first emplaced device $(9.54 \mathrm{KW} / \mathrm{m})$ and nearly 14 -fold improvement over a single coastal device $(11.5 \mathrm{KW} / \mathrm{m})$.

In each case, we see clearly that making use of a series of wave farms spread over the entire ocean basin yields significantly more absorbed energy than placing a single farm at the coastline.

\section{Discussion and concluding remarks}

\subsection{General aspects}

We have set out to show the latent potential for harnessing wave power in deep water. As detailed in the introduction, this allows energy that would otherwise be lost to friction and wave breaking to be utilized over the vast extent of the world's oceans. The field of wave power technology is still rather undeveloped compared with other renewable energy sources (notably wind and solar power), and many technical hurdles remain to be addressed before wave power can become economically competitive. Situating devices in the open oceans adds additional complexities to those faced by current, near-shore devices. Among these are higher installation, maintenance and servicing costs; higher transmission losses (see e.g., the estimation of $9.8 \%$ losses for a 100 MW farm theoretically placed $100 \mathrm{~km}$ offshore in Henfridsson et al. 2007) and longer lengths of undersea cable, which provide a vector for failure; more costly mooring systems; as well as a much harsher wave climate, which may place additional demands on survivability. Nevertheless, while problems of electrical power transmission and farm maintenance are clearly exacerbated for installations far offshore, these problems are partly counteracted by reduced impacts on ecology, morphodynamics and infrastructure of coastal zones (see e.g., the recent reviews of environmental impacts of wave-energy converters, e.g., Langhamer et al. 2010 or Pelc and Fujita 2002). In addition, conflicting interests in terms of fisheries, oil and gas, offshore wind, and others may be significantly reduced offshore (see Schlüt- ter et al. 2015). Of course, the ambition to exploit the more powerful wave regimes and, as we have emphasized, the possibility of using the large expanses of the open seas should be seen as the driving force behind the move farther offshore in the long-term future of wave energy technology.

\subsection{The twin-plate model}

We have presented a new, simple model for a wave power harvesting device in deep water, based on the solution for a single vertically floating plate given by Haskind (1959). This model is analytically tractable and has the advantage of a closed-form solution. In contrast to the two independently floating plates discussed by Srokosz and Evans (1979), based on work on a single plate by Ursell $(1947,1948)$, the design presented herein operates on the basis of power generation from the relative motion of the plates.

It is straightforward to add width and density to each plate, and calculate their masses and moments of inertia; however, the performance of the device is not qualitatively altered thereby. Likewise, the addition of springs, while requiring the specification of additional input parameters, does not increase the value of the two-plate configuration as an illustrative model for obtaining transfer functions. ${ }^{1}$

Although a recent analysis due to Bódai and Srinil (2015) points to the optimality in irregular waves of extreme geometries for box-hull devices with two degrees of freedompointing towards either a wave terminator or attenuator design — as noted in Scher et al. (1983) mooring forces for large, beam-sea absorbers may be prohibitively large. For this and other reasons, we see the proposed twin-plate device primarily as a means to provide frequency-dependent transmission, reflection and absorption coefficients.

With some notable exceptions [e.g., Srokosz and Evans (1979), or the arrays of small buoys treated by Garnaud and Mei (2009) in water of intermediate depth $(k h=O(1)$ for water depth $h$ )], few solutions providing explicit transfer functions seem to be available in the literature. As a result of this relative scarcity of data on device performance, studies of the impacts of wave-energy devices on the subsequent coastal wave climate have had to rely on a number of simplifications; in one of the earliest such studies, Millar et al. (2007) specified explicit, frequency-independent transmission percentages for the converter device. In a later follow-up, Smith et al. (2012) modeled the devices as damped linear oscillators. Other studies, such as that by Palha et al. (2010)

\footnotetext{
1 The 2D geometry imposes a somewhat more severe restriction, in that it does not enable one to take into account oblique incidence and thus the angular distribution of wave spectra. Due to its periodicity along the plate (i.e., orthogonal to the $x-z$ plane), and the infinite extent of the plates, any obliquely incident component will impose zero forces and moments, and the structure will not move.
} 
focused on specific, commercial technology (i.e., Pelamis energy devices).

Thus, we consider the simple solution presented here, which readily yields absorption, reflection, and transmission coefficients as functions of frequency, to serve as an illustrative model when considering the potential of wave power harvesting in the open sea.

\subsection{Fetch-dependent wave energy harvesting}

We take as given the basic fact that the wind transfers energy to the waves only up to a point, after which the sea state may be assumed to be 'fully developed,' and further energy is dissipated by breaking. Thus, for fetches larger than that needed for this saturation, it is clearly of interest to capture the energy multiple times, allowing it to grow under the influence of the wind each time. Within the limitations of our twodimensional model, we have discussed the main mechanisms involved in assessing the promise of such an approach.

Taking the JONSWAP spectrum as input, and essentially without further calculation, we have shown that a series of ideal wave farms - which are assumed to capture the entire energy of the incoming sea state-spaced along a basin of length $2000 \mathrm{~km}$, outperform a single coastal farm by a factor of 5 under a design wind of $U_{10}=10 \mathrm{~m} / \mathrm{s}$. In the same basin, for a wave energy farm represented by the transfer functions derived herein, a series of sequential farms outperforms a single one placed at the coast by a factor of 14 under design conditions. Even with sub-optimal winds, an 11-fold increase is still observed. In addition, some preliminary results are given on the spacing of the wave farms over the basin, subject to the efficiency of the farm and the strength of the wind; this is found to be on the order of $100 \mathrm{~km}$. That is, from a purely physical standpoint, the energy shadow of our theoretical wave farm-which we believe is characterized realistically by the transfer functions we have given-is such that the spacing between subsequent farms should be on the order of $100 \mathrm{~km}$. Economical considerations may, of course, modify this analysis. Taken together, the results support our contention (expressed also by Falnes 2007, using different methods) that the wave power potential on the open ocean is considerably larger than the coastal potential.

The approach we have adopted is, of necessity, simplified, and leaves many technical problems unresolved. There remains room for future work on the performance of largescale, sparse arrays, or farms, of wave-energy converters. It is to be hoped that these will yield transmission, reflection and absorption values to replace the ones derived herein with the help of a twin-plate model. Moreover, it is to be hoped that they will facilitate the consideration of three-dimensional wave spectra, and provide a more comprehensive picture of the promise of open-ocean wave energy extraction.
Acknowledgments This research was supported by the Israel Science Foundation (Grant 464/13). RS was also supported in part at the Technion by a fellowship from the Lady Davis Foundation.

\section{Appendix 1: Mathematical expressions for a single plate, based on Haskind}

The expressions for the coefficients appearing in Eqs. (13), (14), and (15) are as follows:

$$
\begin{aligned}
& t=\frac{-j K_{1}}{\pi I_{1}-j K_{1}} ; \quad r=1-t ; \\
& B_{2}=\frac{-2 j \mu S_{1}}{\pi I_{1}-j K_{1}} ; \quad B_{4}=\frac{-2 j T\left(S_{1}-\pi / 4\right)}{\pi I_{1}-j K_{1}} ; \\
& Y_{g}=\frac{-2 g T S_{1}}{\pi I_{1}-j K_{1}} ; \quad M_{g}=\frac{-2 g T^{2}\left(S_{1}-\pi / 4\right)}{\mu\left(\pi I_{1}-j K_{1}\right)} ; \\
& \lambda_{22}=\frac{4 \omega T^{2} S_{1}^{2}}{\pi^{2} I_{1}^{2}+K_{1}^{2}} ; \quad \lambda_{44}=\frac{4 \omega T^{4}\left(S_{1}-\pi / 4\right)^{2}}{\mu^{2}\left(\pi^{2} I_{1}^{2}+K_{1}^{2}\right)} ; \\
& \lambda_{24}=\lambda_{42}=\frac{4 \omega T^{3} S_{1}\left(S_{1}-\pi / 4\right)}{\mu\left(\pi^{2} I_{1}^{2}+K_{1}^{2}\right)} ; \\
& \mu_{22}=\frac{4 T^{2}}{\pi}\left(\frac{1}{2}-\frac{S_{0}}{\mu}+\frac{S_{0}^{-1}}{\mu^{2}}-\frac{S_{1} \Gamma}{\mu\left(\pi^{2} I_{1}^{2}+K_{1}^{2}\right)}\right) ; \\
& \mu_{24}=\mu_{42}=\frac{4 T^{3}}{\pi}\left(\frac{\pi}{12}+\frac{1}{2 \mu}-\frac{S_{0}}{\mu^{2}}+\frac{S_{0}^{-1}}{\mu^{3}}\right. \\
& \left.-\frac{S_{1} \Gamma-\pi \Gamma_{0} / 4}{\mu^{2}\left(\pi^{2} I_{1}^{2}+K_{1}^{2}\right)}\right) \\
& \mu_{44}=\frac{4 T^{4}}{\pi}\left(\frac{4+\pi^{2}}{8 \mu^{2}}+\frac{\pi}{6 \mu}+\frac{\pi^{2}}{64}-\left(\frac{1}{\mu^{3}}+\frac{\pi}{4 \mu^{2}}\right) S_{0}\right. \\
& \left.+\frac{S_{0}^{-1}}{\mu^{4}}-\frac{\left(S_{1}-\pi / 4\right)\left(\Gamma / \mu-\pi \mu \gamma_{2} / 4\right.}{\mu^{2}\left(\pi^{2} I_{1}^{2}+K_{1}^{2}\right)}\right) \text {. }
\end{aligned}
$$

In the above formulae $\Gamma, \Gamma_{0}$, and $\gamma_{2}$ are given by:

$$
\begin{aligned}
& \Gamma=\gamma_{1}-\mu \gamma_{2}-0.5 \pi K_{1} ; \\
& \quad \Gamma_{0}=\mu^{2} S_{1} \gamma_{2}-\mu S_{0}\left(\pi^{2} I_{1}^{2}+K_{1}^{2}\right) \\
& \gamma_{1}=\pi^{2} I_{o}^{-1} I_{1}-K_{0}^{-1} K_{1} ; \quad \gamma_{2}=\pi^{2} I_{0} I_{1}-K_{0} K_{1}
\end{aligned}
$$

where $S_{0}=0.5 \pi\left(I_{0}+L_{0}\right)$ and $S_{1}=0.5 \pi\left(I_{1}+L_{1}\right) / \mu$.

$L_{0}, L_{1}$ and $K_{0}, K_{1}, I_{0}, I_{1}$ are Struve and Bessel functions of the argument $\mu=k T$, respectively. The terms $S_{0}^{-1}, I_{0}^{-1}$ and $K_{0}^{-1}$ are given by 


$$
\begin{aligned}
& S_{0}^{-1}=\int_{0}^{\mu} S_{0}(\xi) \mathrm{d} \xi, \quad I_{0}^{-1}=\int_{0}^{\mu} I_{0}(\xi) \mathrm{d} \xi, \\
& K_{0}^{-1}=\int_{0}^{\mu} K_{0}(\xi) \mathrm{d} \xi .
\end{aligned}
$$

\section{Appendix 2: Design configuration for the illustrative model}

We return to the calculations of Sect. 3.4. Choosing a design wave with $k=k_{\mathrm{p}}$ leaves us with three dimensionless free parameters: $X=k_{\mathrm{p}} T, Y=k_{\mathrm{p}} C$, and $Z=g^{-1 / 2} k_{\mathrm{p}}^{3 / 2} d$. Searching this 3-dimensional parameter space for the maximum value of the dimensionless absorbed power $P_{\mathrm{a}} / F_{\mathrm{i}}$, see (28), gives $P_{\mathrm{a}} / F_{\mathrm{i}}=0.5$ along a curve, as shown in Fig. 7. For all further calculations we have chosen the design triad $T_{m}=0.71 k_{\mathrm{p}}^{-1}, C_{m}=1.05 k_{\mathrm{p}}^{-1}$, and $d_{m}=0.88 g^{1 / 2} k_{\mathrm{p}}^{-3 / 2}$, corresponding to $(X, Y, Z)=(0.71,1.05,0.88)$ as marked on Fig. 7, but other choices are possible.

In Fig. 8 we present this power absorption ratio $P_{\mathrm{a}} / F_{\mathrm{i}}$, as well as the transmitted power ratio $\left|T_{\mathrm{t}}\right|^{2}$ and the reflected power ratio $\left|T_{\mathrm{r}}\right|^{2}$ as functions of the wave number. (This is an extended version of Fig. 5, whose range is depicted in the shaded region.) For the design wave $k / k_{\mathrm{p}}=1$ we have $P_{\mathrm{a}} / F_{\mathrm{i}}=0.5$ and $\left|T_{\mathrm{t}}\right|^{2}=\left|T_{\mathrm{r}}\right|^{2}=0.25$.

The power absorption ratio $P_{\mathrm{a}} / F_{\mathrm{i}}$ drops rather quickly for waves longer than the design wave, and significantly slower for waves shorter than the design wave. The values of the

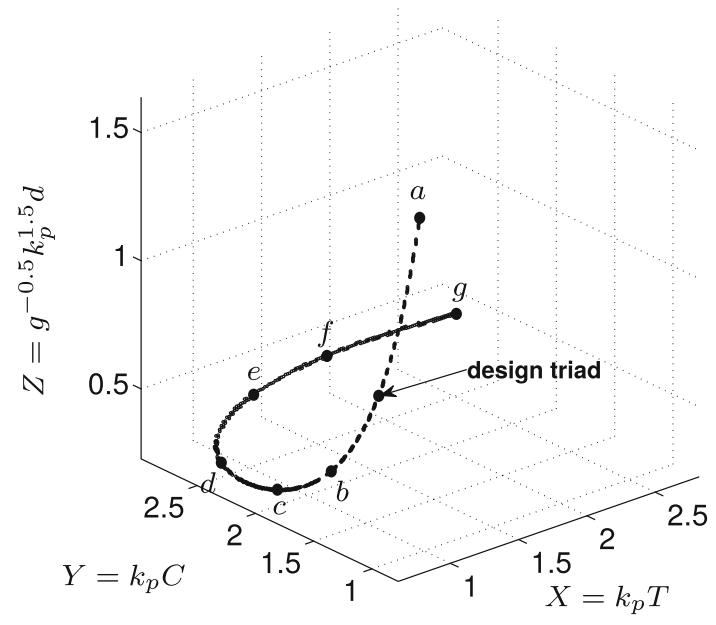

Fig. 7 Curve of maximal power absorption in $(T, C, d)$ parameter space. The values of $(X, Y, Z)$ at the labeled points are: $a=(0.77,0.78,1.61), b=(0.67,1.42,0.51)$, $c=(0.69,1.95,0.32), d=(0.83,2.54,0.26), e=(1.45,2.98,0.33)$, $f=(1.96,2.96,0.39), g=(2.77,2.78,0.44)$

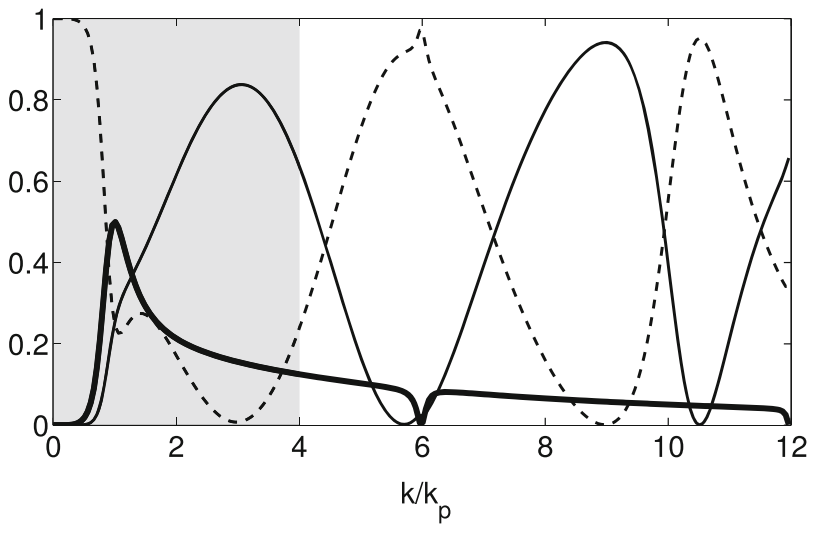

Fig. 8 Power absorption ratio $P_{\mathrm{a}} / F_{\mathrm{i}}$ (thick solid line), power reflection ratio $\left|T_{\mathrm{r}}\right|^{2}$ (thin solid line), and power transmission ratio $\left|T_{\mathrm{t}}\right|^{2}$ (dashed line) as a function of wavenumber for a device with $k_{\mathrm{p}} T=0.71, k_{\mathrm{p}} C=$ $1.05, g^{-1 / 2} k_{\mathrm{p}}^{3 / 2} d=0.88$. The shaded area is depicted in detail in Fig. 5

absorbed power for $k / k_{\mathrm{p}}=0.71$ and 4 are $P_{\mathrm{a}} / F_{\mathrm{i}}=0.1403$ and 0.125 , respectively.

\section{References}

Bódai T, Srinil N (2015) Performance analysis and optimization of a box-hull wave energy converter concept. Renew Energy 81:551565

Cruz J (ed) (2008) Ocean wave energy: current status and future perspectives. Springer, Berlin

Falcão AFO (2010) Wave energy utilization: a review of the technologies. Renew Sustain Energy Rev 14:899-918

Falnes J (2007) A review of wave-energy extraction. Mar Struct 20(4):185-201

Garnaud X, Mei CC (2009) Wave-power extraction by a compact array of buoys. J Fluid Mech 635:389-413

Haskind MD (1959) The radiation and diffraction of surface waves from a vertically floating plate. Prik1 Mat Mekh 23(3):546-556

Hasselmann K, Barnett TP, Bouws E, Carlson H, Cartwright DE, Enke K, Ewing JA, Gienapp H, Hasselmann DE, Kruseman P, Meerburg A, Müller P, Olbers DJ, Richter K, Sell W, Walden H (1973) Measurements of wind-wave growth and swell decay during the Joint North Sea Wave Project (JONSWAP). Deutsch Hydrogr Z Suppl A 8(12):1-95

Henfridsson U, Neimane V, Strand K, Kapper R, Bernhoff H, Danielsson O, Leijon M, Sundberg J, Thorburn K, Ericsson E, Bergman $\mathrm{K}$ (2007) Wave energy potential in the Baltic Sea and the Danish part of the North Sea, with reflections on the Skagerrak. Renew Energy 32(12):2069-2084

Holthuijsen LH (2008) Waves in oceanic and coastal waters. Cambridge University Press, Cambridge

Langhamer O, Haikonen K, Sundberg J (2010) Wave power-sustainable energy or environmentally costly? A review with special emphasis on linear wave energy converters. Renew Sustain Energy Rev 14(4):1329-1335

Magagna D, MacGillivray A, Jeffrey H, Hanmer C, Raventos A, Badcock-Broe A, Tzimas E (2014) Wave and tidal energy strategic technology agenda. SI Ocean, p 44

Miles JW (1957) On the generation of surface waves by shear flows. J Fluid Mech 3:185-204 
Millar DL, Smith HCM, Reeve DE (2007) Modelling analysis of the sensitivity of shoreline change to a wave farm. Ocean Eng 34:884901

Multon B (ed) (2011) Marine renewable energy handbook. Wiley-ISTE, Hoboken

Palha A, Mendes L, Fortes CJ, Brito-Melo A, Sarmento A (2010) The impact of wave energy farms in the shoreline wave climate: Portuguese pilot zone case study using Pelamis energy wave devices. Renew Energy 35:62-77

Pelc R, Fujita RM (2002) Renewable energy from the ocean. Mar Policy 26(July):471-479

Pierson WJ, Moskowitz L (1964) A proposed spectral form for fully developed wind seas based on the similarity theory of S. A. Kitaigorodskii. J Geophys Res 69(24):5181-5190

Plant WJ (1982) A relationship between wind stress and wave slope. J Geophys Res Oceans 87(C3):1961-1967

Rascle N, Ardhuin F (2013) A global wave parameter database for geophysical applications. Part 2: model validation with improved source term parameterization. Ocean Model 70:174-188

Rascle N, Ardhuin F, Queffeulou P, Croizé-Fillon D (2008) A global wave parameter database for geophysical applications. Part 1: wave-current-turbulence interaction parameters for the open ocean based on traditional parameterizations. Ocean Model 25:154-171

Scher RM, Troesch AW, Zhou G (1983) The experimental and theoretical evaluation of a twin-flap wave-energy absorbing device. Ocean Eng 10(5):325-345
Schlütter F, Petersen OS, Nyborg L (2015) Resource mapping of wave energy production in Europe. In: Proceedings of the 11th European wave and tidal energy conference, 10B4-3

Smith HCM, Pearce C, Millar DL (2012) Further analysis of change in nearshore wave climate due to an offshore wave farm: an enhanced case study for the Wave Hub site. Renew Energy 40(1):51-64

Srokosz MA, Evans DV (1979) A theory for wave-power absorption by two independently oscillating bodies. J Fluid Mech 90:337-362

Stiassnie M (1980) A simple mathematical model of a floating breakwater. Appl Ocean Res 2(3):107-111

Stiassnie M, Agnon Y, Naheer E (1981) Scattering of water waves by a system of vertically floating plates. In: International symposium on hydrodynamics in ocean engineering, Trondheim, Norway, pp $1111-1132$

Stiassnie M, Naheer E, Boguslavsky I (1984) Energy losses due to vortex shedding from the lower edge of a vertical plate attacked by surface waves. Proc R Soc A Math Phys Eng Sci 396:131-142

Ursell F (1947) The effect of a fixed vertical barrier on surface waves in deep water. Math Proc Camb Philos Soc 43(03):374-382

Ursell F (1948) On the waves due to the rolling of a ship. Q J Mech Appl Math 1:246-252 